\title{
Development and validation of a nomogram for predicting prostate cancer in men with prostate-specific antigen grey zone based on retrospective analysis of clinical and multi-parameter magnetic resonance imaging/transrectal ultrasound fusion-derived data
}

\author{
Zhimin Ding ${ }^{1 \#}$, Huaiyu Wu ${ }^{1 \#}$, Di Song ${ }^{1}$, Hongtian Tian ${ }^{1}$, Xiuqin $\mathrm{Ye}^{1}$, Weiyu Liang ${ }^{1}$, Yang Jiao ${ }^{1}$, Jintao $\mathrm{Hu}^{2}$, \\ Jinfeng $\mathrm{Xu}^{1}$, Fajin Dong ${ }^{1}$ \\ ${ }^{1}$ Department of Ultrasound, The Second Clinical Medical College, Jinan University, The First Affiliated Hospital, Southern University of Science \\ and Technology (Shenzhen People's Hospital), Shenzhen Medical Ultrasound Engineering Center, Shenzhen, China; ${ }^{2}$ Department of Pathology, The \\ Second Clinical Medical College, Jinan University, The First Affiliated Hospital, Southern University of Science and Technology (Shenzhen People's \\ Hospital), Shenzhen, China \\ Contributions: (I) Conception and design: Z Ding, H Wu; (II) Administrative support: J Xu, F Dong; (III) Provision of study materials or patients: Z \\ Ding; (IV) Collection and assembly of data: Z Ding, H Wu; (V) Data analysis and interpretation: H Wu; (VI) Manuscript writing: All authors; (VII) \\ Final approval of manuscript: All authors. \\ \#These authors contributed equally to this work and shared the first authorship. \\ Correspondence to: Jinfeng Xu, MD; Fajin Dong, MD. Department of Ultrasound, The Second Clinical Medical College, Jinan University, The First \\ Affiliated Hospital, Southern University of Science and Technology (Shenzhen People's Hospital), Shenzhen Medical Ultrasound Engineering \\ Center, Shenzhen, China. Email: xujinfeng@yahoo.com; dongfajin@szhospital.com.
}

Background: Urologists face a dilemma when deciding whether prostate biopsy is required for patients with prostate-specific antigen (PSA) levels in the grey zone (4 to $10 \mathrm{ng} / \mathrm{mL}$ ).

Methods: We retrospectively analyzed data from consecutive patients with PSA levels in grey zone, who underwent targeted multiparametric magnetic resonance imaging (MP-MRI)/transrectal ultrasound (TRUS) fusion biopsy with elastography between November 2017 and December 2019 in our hospital. The patientse data including age, PSA, fPSA (free PSA), fPSA/PSA, PSA density (PSAD), prostate volume, elastography Q-analysis score (EQS), and prostate imaging-reporting and data system (PI-RADS) score were collected. The nomogram was built using logistic regression and the final cohort of patients was randomly divided into a training cohort (70\%) and a validation cohort (30\%) by $\mathrm{R}$ software. The models were evaluated by receiver operating characteristic curve (ROC) analysis and calibration curve analysis. The nomogram was constructed from the best model.

Results: The final study cohort consisted of 155 patients (training cohort, 109 patients; validation cohort, 46 patients) with PSA in the grey zone, of which 36 patients were pathologically diagnosed with PCa. The EQS model, -EQS model, +EQS model were built. The +EQS model that consisted of fPSA/PSA, EQS, and PI-RADS score had the best PCa diagnostic accuracy (development and validation, 0.783 and 0.781 ) and probability score (development and validation, 0.939 vs. 0.622 ). The new nomogram based on this model was constructed, in which fPSA/PSA ratio had the largest impact, followed by PI-RADS and EQS.

Conclusions: Elastography and pre-biopsy MP-MRI has clinical significance for patients with PSA in the grey zone. The new nomogram, which is based on pre biopsy data including serological analysis, PI-RADS score, and EQS, can be helpful for clinical decision-making to avoid unnecessary biopsy.

Keywords: Prostate cancer (PCa); prediction model; multi-parameter magnetic resonance imaging; elastography; image fusion

Submitted Jul 12, 2020. Accepted for publication Sep 25, 2020.

doi: $10.21037 /$ tau-20-1154

View this article at: http://dx.doi.org/10.21037/tau-20-1154 


\section{Introduction}

Prostate cancer $(\mathrm{PCa})$ is the most commonly diagnosed cancer and the second highest leading cause of cancer mortality in men $(1,2)$. The prostate-specific antigen (PSA) blood test is widely used as a PCa screening tool $(1,3)$; a serum PSA level higher than $4 \mathrm{ng} / \mathrm{mL}$ necessitates biopsy (4). However, only $26 \%$ of men whose PSA levels are in the "grey zone" (4 to $10 \mathrm{ng} / \mathrm{mL}$ ) are diagnosed with PCa (5). Therefore, large numbers of patients suffer through unnecessary biopsies (6-8). Urologists face a dilemma when deciding whether biopsy is required for patients with PSA levels in the grey zone (referred to as grey zone patients).

Studies have shown that multiparametric magnetic resonance imaging (MP-MRI)/transrectal ultrasound (TRUS) fusion biopsy has good prospects for clinical application in targeted prostate biopsy (9-12); it combines the advantages of MRI as an effective imaging method to detect $\mathrm{PCa}$ (13), with the real-time dynamic characteristics of TRUS and its ability to position the target to achieve precise biopsy sampling. In a study that included 39 grey zone patients, researchers found that MP-MRI/TRUS fusion targeted biopsy improved the rate of PCa diagnosis (14). However, PCa risk prediction was not assessed due to the small sample size. Niu et al. proposed an effective prediction model to diagnose high-grade prostate cancer (HGPCa) in grey zone patients based on MP-MRI (15); but the study included only one imaging method (MRI) and the result was not externally validated. Fang et al. proposed a 'prostate volume + age + magnetic resonance imaging + digital rectal examination (PAMD)' score based on 345 grey zone cases, and the model showed good predictive accuracy for HGPCa (16). Other prediction models for grey zone patients have been proposed in Japan, USA, and Korea (17-19). Although MRI-based prediction models in the above studies (15-19) have shown good PCa predictive ability for PSA grey zone patients, the modalities have some limitations (20), such as lower specificity; benign lesions, such as prostatitis, scarring, high-grade prostatic intraepithelial neoplasia, and hyperplasia, display PCa characteristics on MRI. In addition, there could be inter-observer inconsistencies in MRI (21-23).

Numerous studies have shown that ultrasound (US) elastography can improve the accuracy of PCa diagnosis (24-29), by evaluating the stiffness of the lesion (30) and guiding the targeted biopsy $(29,31)$. To date, a prediction model that combines lesion elasticity with MP-MRI and clinical data in men with PSA grey zone levels has not been reported.

Our study aims to develop and validate a diagnostic prediction model for $\mathrm{PCa}$ to be used in conjunction with pre-biopsy MP-MRI/TRUS fusion, based on clinical indicators, MP-MRI, elastography and other possible risk factors. This would reduce the physical and psychological burden on patients, and the reduction in unnecessary medical procedures would bring down the cost of healthcare, benefitting society at large.

We present the following article in accordance with the TRIPOD reporting checklist (available at http://dx.doi. org/10.21037/tau-20-1154).

\section{Methods}

\section{Patient selection}

This retrospective study was approved by the institutional review board of Shenzhen People's Hospital, and all patients provided informed consent. The study was conducted in accordance with the Declaration of Helsinki (as revised in 2013). In the initial cohort, we included consecutive patients who underwent prostate biopsy in department of ultrasound from November 2017 to December 2019. Patients with incomplete or ambiguous clinical data were excluded, e.g., outside the grey zone, no age, no fPSA, no targeted MPMRI/TRUS fusion biopsy, no EQS. The final cohort of 155 patients was randomly divided into a training cohort (70\%) and a validation cohort (30\%) by $\mathrm{R}$ software. The inclusion criteria were as follows: serum PSA level in grey zone (4 to $10 \mathrm{ng} / \mathrm{mL}$ ), available pathological results, and no other prior treatment.

\section{Multiparametric magnetic resonance imaging}

MP-MRI was acquired by 3.0 T Skyra ultra-high field magnetic resonance scanner (Siemens Germany), using 18-channel body matrix coil. The patients were instructed to urinate a small amount, and were scanned approximately $1 \mathrm{~cm}$ above the pubic symphysis. The scanning range included at least the prostate and the seminal vesicle. Imaging included T2-weighted imaging (T2WI) sequence (including the cross section, sagittal plane, and coronal plane), diffusion-weighted imaging (DWI) generated by single-shot spin echo planar imaging (SS-EPI) sequence, and dynamic contrast-enhanced MRI (DCE-MRI) was generated by fat-suppressed T1-weighted imaging (T1WI) sequence with $3 \mathrm{D}$ spoiled gradient echo, after rapid 
intravenous injection with gadopentetate dimeglumine $(0.2 \mathrm{mmol} / \mathrm{kg})$.

An experienced radiologist blinded to patient clinical manifestations and auxiliary diagnostic results reported their evaluations using the prostate imaging-reporting and data system version 2 (PI-RADS v2) (32). Based on the apparent diffusion coefficient (ADC) map and T2WI, the suspicion levels of PCa with clinical significance was scored from I to $\mathrm{V}$ as follows: (I) extremely unlikely to be malignant; (II) unlikely to appear; (III) ambiguous; (IV) malignancy possibly exists; and (V) malignancy likely exists. A maximum of two index lesions were chosen per each patient. Only $\mathrm{ADC}$ maps were used for image registration, regardless of the location of the index lesion.

\section{Ultrasound}

The GE Logiq E9 ultrasound system (GE Healthcare, Milwaukee, USA) with volume navigation and elastography functions was used. Patients underwent an enema cleansing before the examination. The left lateral position was adopted, and the hip and the knees were bent. Patients were instructed to relax and take a deep breath. The probe was inserted gently into the anus. First, the size of the prostate was measured. The operator then focused on whether the shape of the prostate was regular, whether the outline was smooth or rough, and whether an abnormal echo area was present. The blood flow status was evaluated using color Doppler flow imaging, with a focus on determining the presence or absence of focal blood-rich areas.

\section{Volume navigation}

Volume navigation, or the fusion imaging technique, used an electromagnetic field tracking system with an electromagnetic transmitter adjacent to the patient, and electromagnetic sensors, or trackers, attached to the US transducer. A fully integrated position sensor unit installed in the US machine received the location data from the sensors. Prior to examination, the axial MP-MRI DICOM files were uploaded to the US machine. Image registration between the MP-MRI and US images was necessary in order to fuse both images correctly. Image registration consisted of plane-to-plane registration, followed by pointto-point registration. All registrations were performed in the axial plane. First, we used muscoli puborectalis as the primary plane for registration; then, based on patients' conditions, different points such as cysts and urethral orifices were selected. Subsequently, the MR images of the reference plane and selected points were matched in the overlay mode to increase observational accuracy. After image registration, the images obtained from TRUS and MRI were displayed in a side-by-side format (Figure 1A) and overlay status (Figure 1B).

\section{Elastography}

Elastographic mode was used to examine suspicious lesions discovered by volume navigation. The region of interest (ROI) was defined as the half side of the gland where the lesion was located $(28,29)$. A dual-display screen was used to simultaneously observe grayscale ultrasound and elastic images (Figure 1C). The suspicious lesion was displayed in the center of the screen and vertical targeted pressure was applied. The dynamic elastography images were stored and analyzed with the elastographic Q-analysis score (EQS) software preset on the equipment. The EQS analysis curve was kept straight as far as possible (fluctuation range of less than 1), to standardize the quality of image. EQS analysis software was used for calculating the mean EQS (Figure 1D).

\section{Prostate biopsy}

The targeted biopsy was performed with a GE Logiq E9 US machine equipped with a 7-9 $\mathrm{MHz}$ multi-frequency IC5-9-D endocavitary probe by the same sonographer who had conducted the MP-MRI/TRUS image fusion. Local anesthesia (lidocaine, $10 \mathrm{~mL}$ ) was administered before each biopsy. The probe was equipped with a $5^{\circ}$ puncture trestle. An 18-gauge, $20 \mathrm{~cm}$ automatic cutting needle and an automated biopsy gun (ACECUT, TSK Laboratory, Tochigi, Japan) were used. Under the guidance of the fused MRI-US images, two biopsy cores per suspicious lesion were sampled (Figure 1E). After targeted biopsies, the systematic prostate biopsy sampling twelve cores was performed; this included obtaining from each side of the gland two cores from the base, two cores from the middle, and two cores from the apex. The reference standard was core-based biopsy pathologic results. The Gleason score (GS) for each core was reported by a genitourinary pathologist who was blinded to the clinical results (Figure 1F).

\section{Study variables and statistical analysis}

The following clinical, MP-MRI and US variables were included in this study: age, PSA, fPSA (free PSA), fPSA/ 

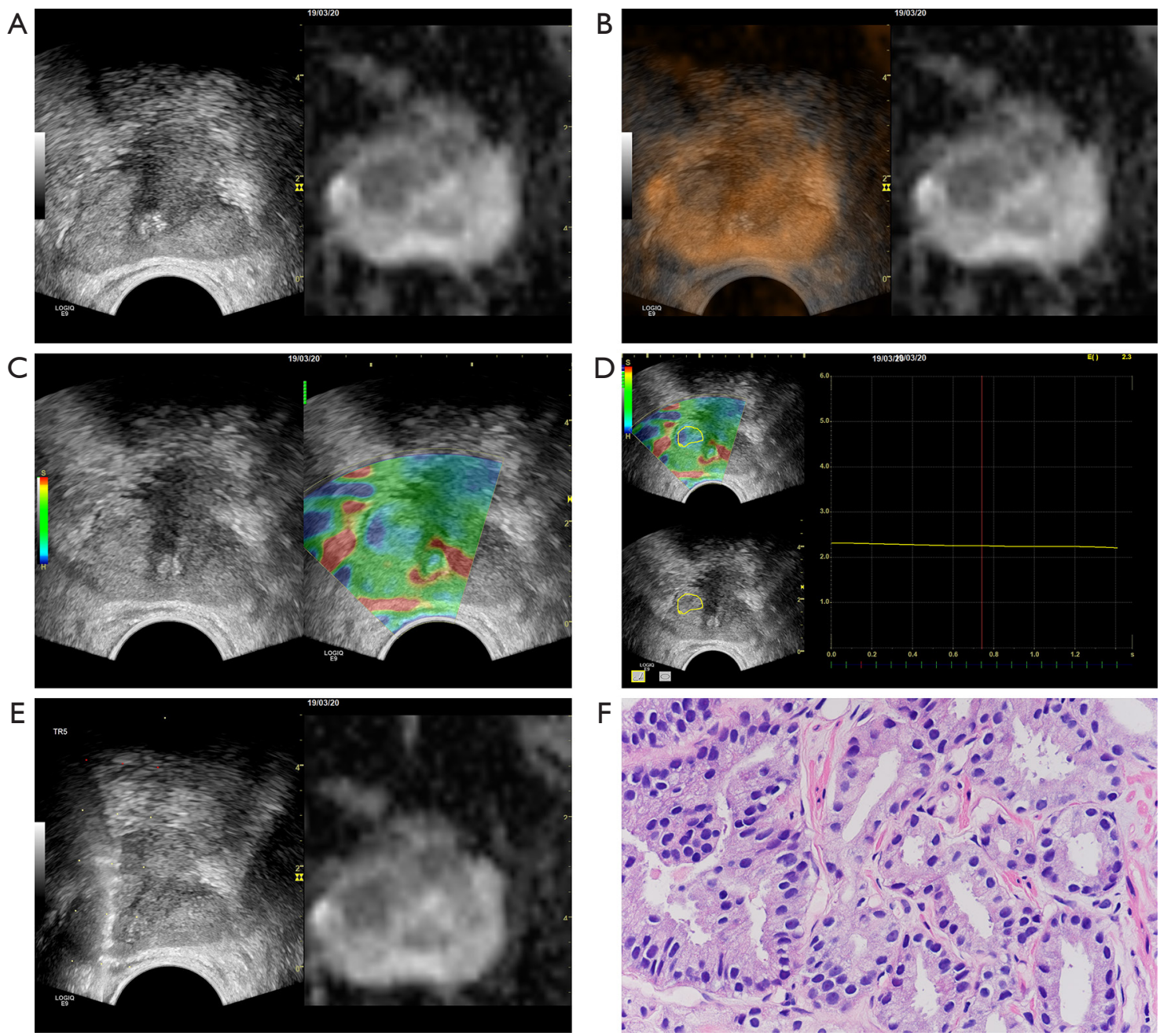

Figure 1 A 60 -year-old patient $\left(\mathrm{PSA}=6.09 \mathrm{ng} / \mathrm{mL}\right.$, fPSA $=0.48 \mathrm{ng} / \mathrm{mL}, \mathrm{fPSA} / \mathrm{PSA}=0.079$, TRUS-derived prostate volume $=20.8 \mathrm{~cm}^{3}$, PSAD $=0.29 \mathrm{ng} / \mathrm{mL} / \mathrm{cm}^{3}$ ) had a pre-biopsy MRI that detected an abnormal signal area of about $14 \mathrm{~mm} \times 10 \mathrm{~mm}$ at the right transition zone of the prostate; the PI-RADS score was 4. (A) Image registration of ADC-US fusion; (B) the overlay image of the ADC-US fusion; (C) elastography detected the suspicious zone with increased stiffness, which was previously detected by MRI; (D) the EQS of the suspicious zone was 2.3; (E) targeted biopsy of the lesion detected by MRI and elastography; (F) targeted biopsy result was positive for PCa (HE staining, $\times 40)$, with a GS of $7(3+4)$ and classified as group 2 according to the WHO/ISUP classification system.

PSA, PSA density (PSAD) calculated by PSA divided by TRUS-derived prostate volume, TRUS-derived prostate volume, EQS, PI-RADS score, and GS. The TRUSderived prostate volume was calculated by multiplying 0.52 with the diameters of the prostate gland obtained from three different axes (up-down, left-right, and front-rear). In the case of multiple PSA tests, the result closest to the biopsy date was used. If the patient had multiple lesions, the highest GS was used. Patients with incomplete reports were excluded from the study.
Statistical analysis was performed using RStudio v1.1 (SAS Institute, Inc., Cary, USA). The significance level was set at $\mathrm{P}=0.05$. A normality test was performed for each variable. If it followed a normal distribution, it was represented by mean \pm standard deviation (SD). If not, the median and the interquartile range (IQR) were reported.

The optimal variables from the training cohort were selected by least absolute shrinkage and selection operator (LASSO) regression and cross-validation. LASSO is the most famous method for analyzing survival data, also 


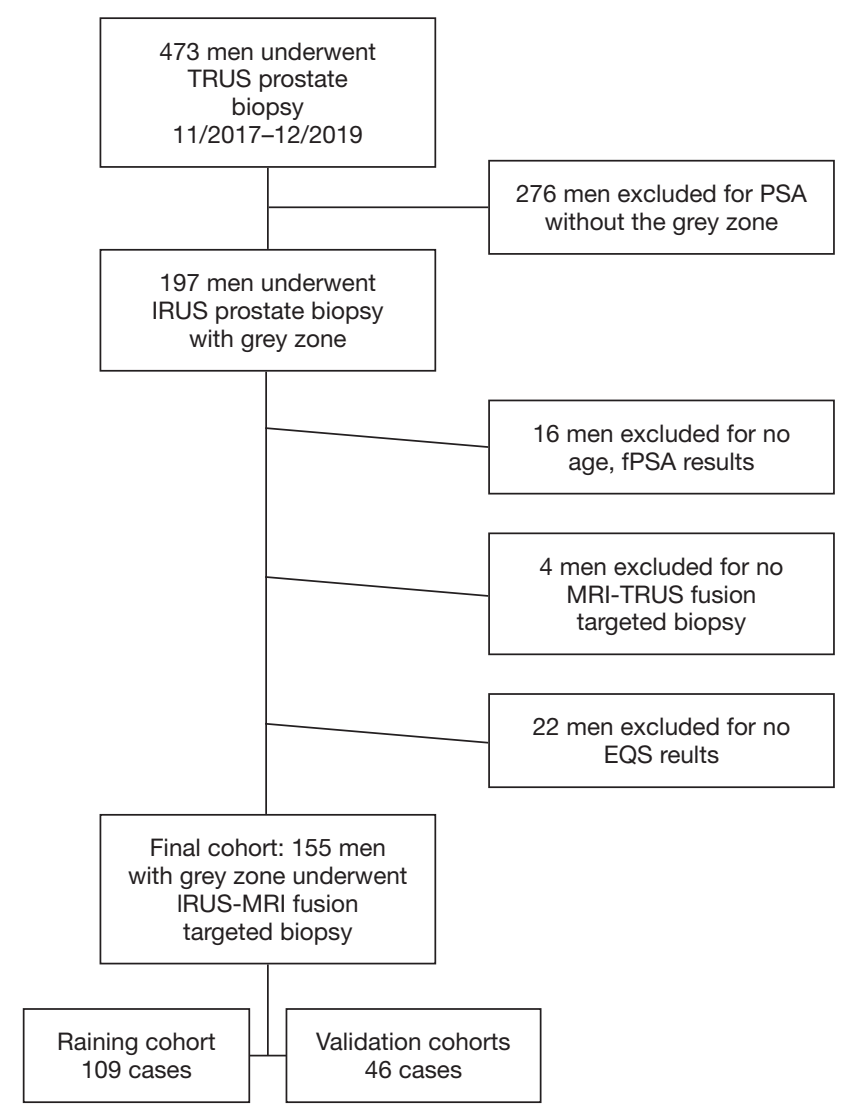

Figure 2 Study flow chart.

suitable in logistic regression $(33,34)$. We constructed LASSO regression models using the glmnet $\mathrm{R}$ package. The value of the tuning parameter (or, penalization coefficient) $\lambda$ of the generated model was negatively associated with the complexity of the model and the value of deviance. When the value of the $\lambda$ increased from left to right, the number of non-zero coefficients increased accordingly. The optimal value of $\lambda$ (that is, the minimum average binomial deviance) was determined by 10 -fold cross-validation via minimum criteria. This value was then used to select the optimal clinical predictors.

Using these predictors with age, PSA, fPSA, fPSA/PSA, PSA density (PSAD), TRUS-derived prostate volume, EQS, and PI-RADS score, multiple logistic regression analysis was performed to generate prediction models. The logistic regression model was adopted to determine a patient-specific clinical signature, which is a linear combination of selected features with their respective weights. Next, the clinical signature was evaluated for its diagnostic ability and calibrated using both the training and validation cohorts. Diagnostic ability was determined by the area under the ROC curve (AUC) values of the receiver operating characteristic (ROC) curves constructed for each model. Calibration, the degree of concordance between the probability predicted by the model and the observed probability in our sample, was examined by plotting the observed values against the predicted values. The apparent trend was analyzed by both linear logistic regression and non-parametric (LOESS) regression, the latter of which allows for any non-linearity in the trend (35).

\section{Nomogram}

A nomogram was constructed from the optimal model, using the R package "rms". The nomogram is used by locating a patient's position for each variable on the horizontal scale. A point value is assigned according to the point scale (top axis) and summed for all variables. The total points correspond to a probability value for having PCa.

\section{Results}

\section{Selection of diagnostic parameters}

A total of 473 patients who had undergone prostate biopsy were initially included in this study (Figure 2). After applying the exclusion criteria, the final study cohort consisted of 155 patients with PSA in the grey zone. The baseline clinical characteristics of these patients are presented in Table 1. The final cohort was further divided into a training cohort (109 cases) and a validation cohort (46 cases). The data on the following 8 clinical characteristics were extracted from the final cohort: age, PSA, fPSA, fPSA/ PSA, PSAD, TRUS-derived prostate volume, EQS, and PI-RADS score. The 10-fold cross-validation yielded the optimal value of $\lambda=0.03939082$, and thus $\log (\lambda)=-3.234222$ (Figure $3 A$ ). The subsequent LASSO regression analysis resulted in three non-zero features (Figure 3B), namely fPSA/PSA, PI-RADS and EQS.

\section{Models}

Multivariate logistic regression analysis revealed that fPSA/PSA, EQS, and PI-RADS score were independent predictors of $\mathrm{PCa}$ in grey zone patients (Table 2); we named this the "EQS model" (Figure 4). In addition, in order to verify the necessity of EQS for PCa prediction, we created the following two models: the "EQS model" that depended 


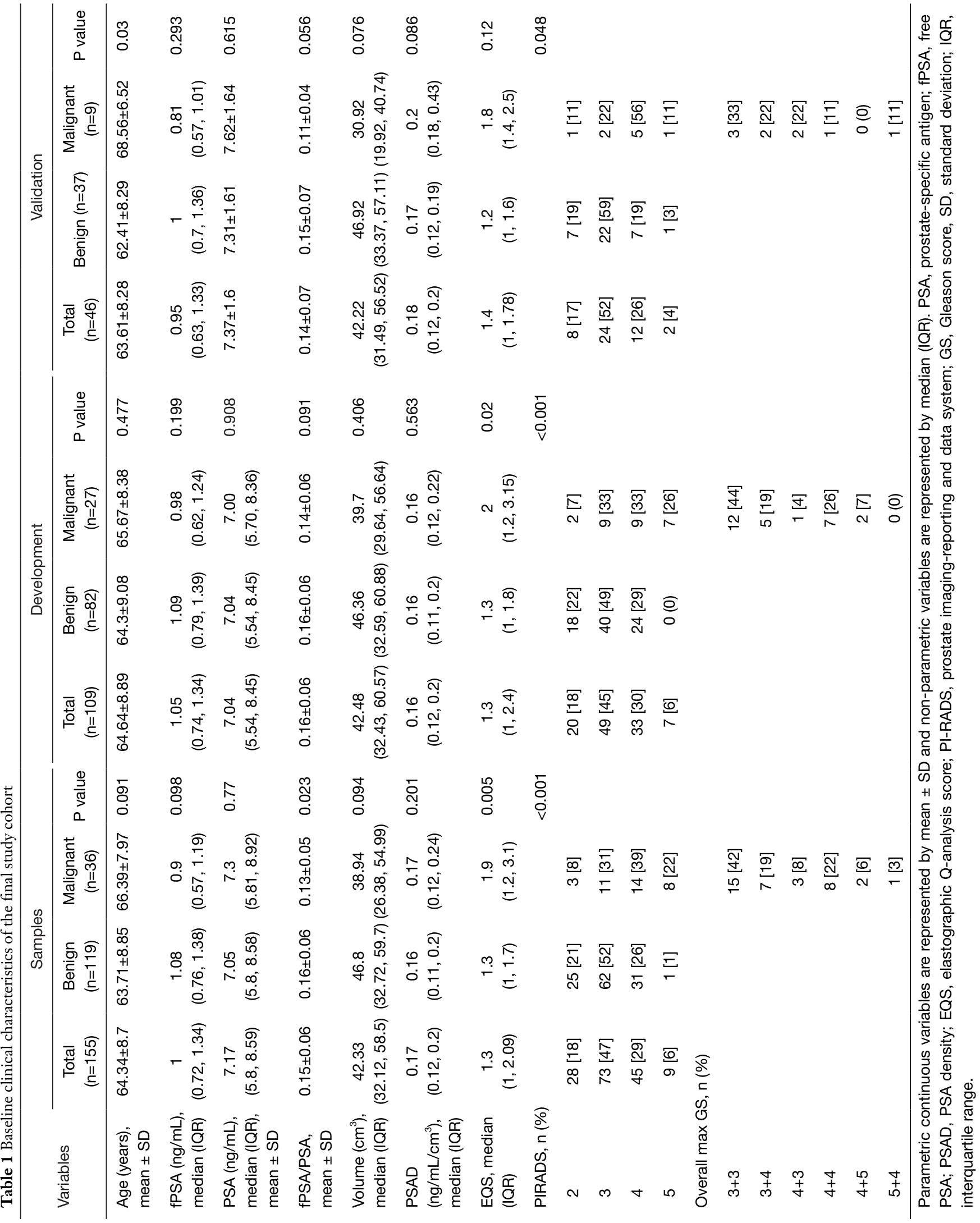



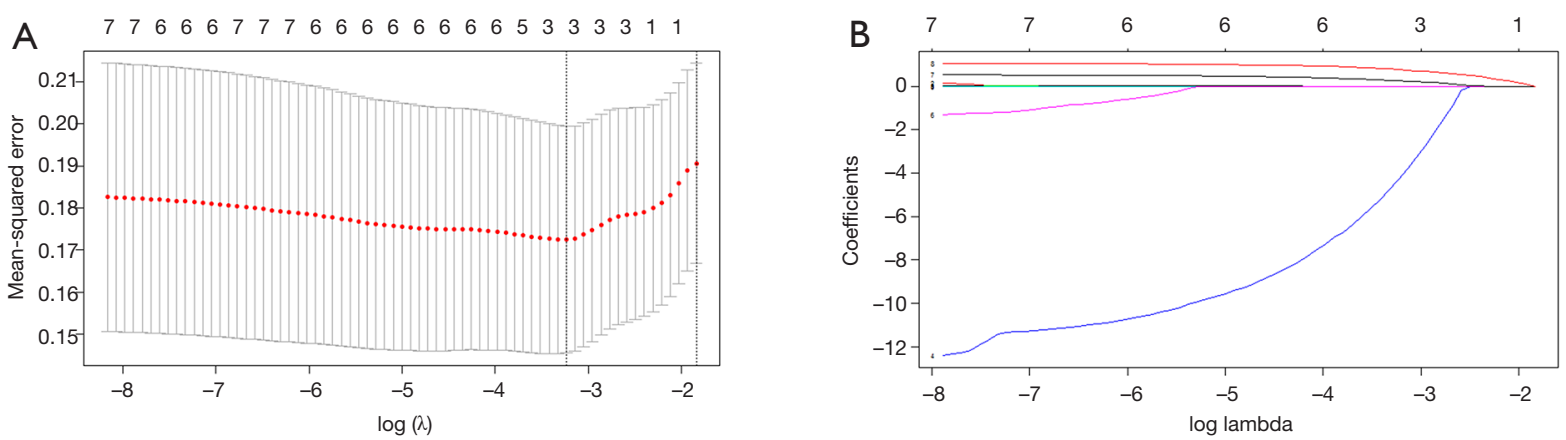

Figure 3 The 10-fold cross-validation and LASSO regression to select the optimal variables for prostate cancer prediction model for grey zone patients. Lambda: $\lambda$. (A) The 10 -fold cross-validation process was repeated to select the optimal penalization coefficient $\lambda$. The value of $\lambda$ yielded the minimum average binomial deviance that was used to select features; (B) as a result, LASSO coefficient profile plot of the 8 features against $\log (\lambda)$.

Table 2 Multivariate logistic regression modeling for predicting prostate cancer

\begin{tabular}{lll}
\hline Model & OR $(95 \% \mathrm{Cl})$ & P value \\
\hline +EQS model & $0.01(0.00,0.15)$ & 0.001 \\
$\begin{array}{l}\text { (Intercept) } \\
\text { fPSA/PSA }\end{array}$ & $0.00(0.00,0.14)$ & 0.026 \\
EQS & $1.70(1.03,2.87)$ & 0.040 \\
PI-RADS score & $3.09(1.64,6.32)$ & 0.001 \\
EQS model & & \\
(Intercept) & $0.11(0.04,0.29)$ & $<0.001$ \\
EQS & $1.78(1.15,2.79)$ & 0.010 \\
-EQS model & & \\
(Intercept) & $0.02(0.00,0.19)$ & 0.002 \\
fPSA/PSA & $0.00(0.00,0.50)$ & 0.044 \\
PI-RADS score & $3.42(1.86,6.89)$ & $<0.001$ \\
\hline
\end{tabular}

OR, odds ratio; $\mathrm{Cl}$, confidence interval; PSA, prostate-specific antigen; fPSA, free PSA; EQS, elastographic Q-analysis score; PI-RADS, prostate imaging-reporting and data system.

solely on EQS, and the "-EQS model", which depended on fPSA/PSA and PI-RADS score (Table 2) (Figures 5,6).

\section{Model development}

The cut-off values of the 3 models were determined based on the ROC curve with consideration to a tradeoff between diagnostic sensitivity and specificity. The cut-off values of the +EQS model, EQS model, -EQS model are
$0.178,0.210$, and 0.219 , respectively; and their AUC values (measurement of diagnostic accuracy) are $0.783,0.649$, and 0.760 , respectively (Figures $4 A, 5 A, 6 A$ ). The calibration plots of the models using the training cohort showed that the probability estimates (p) for accurate $\mathrm{PCa}$ diagnosis for the +EQS model, EQS model, -EQS model were 0.939, 0.997, and 0.921 , respectively (Figures 4B,5B,6B), indicating good agreement with clinical findings.

\section{Model validation}

Using data from the validation cohort, we performed ROC curve analysis for our three models. The cut-off values of +EQS model, EQS model, -EQS model were found to be $0.318,0.238$, and 0.253 , respectively, and their AUC values were $0.781,0.670$, and 0.790 , respectively (Figures $4 C, 5 C, 6 C$ ). The $-\mathrm{EQS}$ model and +EQS model demonstrated good diagnostic accuracy. The calibration of the models revealed that +EQS model had the highest probability for diagnosing $\mathrm{PCa}(\mathrm{P}=0.622$; Figure $4 D)$; followed by the EQS model ( $\mathrm{P}=0.608$; Figure $5 D)$, and the EQS model had the lowest probability $(\mathrm{P}=0.368$; Figure $6 D)$. Overall, +EQS model has the best ability to predict $\mathrm{PCa}$ in grey zone patients.

\section{Nomogram}

We constructed a new nomogram based on the +EQS model (fPSA/PSA + EQS + PI-RADS score; Figure 7). Within the model, fPSA/PSA ratio had the largest impact, followed by PI-RADS score, and finally EQS. Lower fPSA/ PSA ratio, higher PI-RADS score, and higher EQS were 

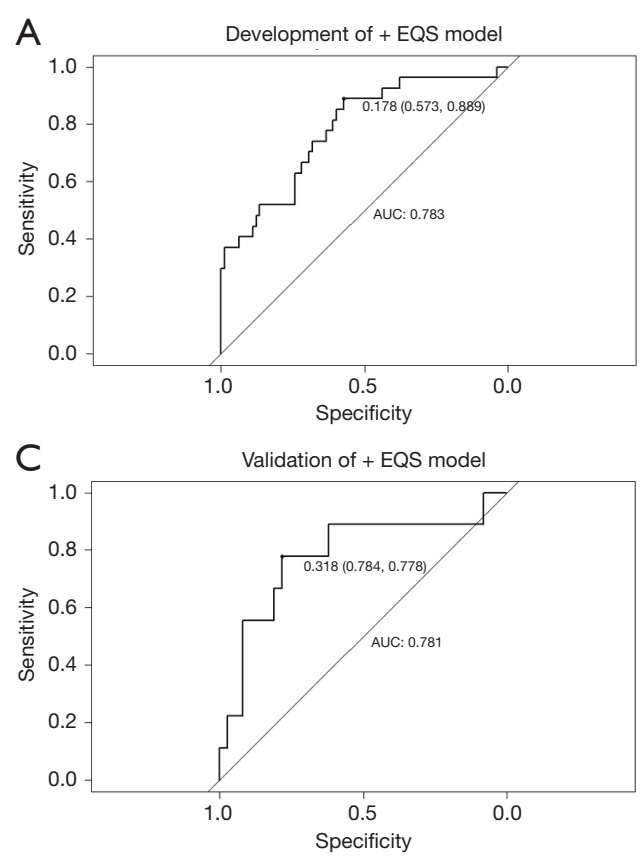

B

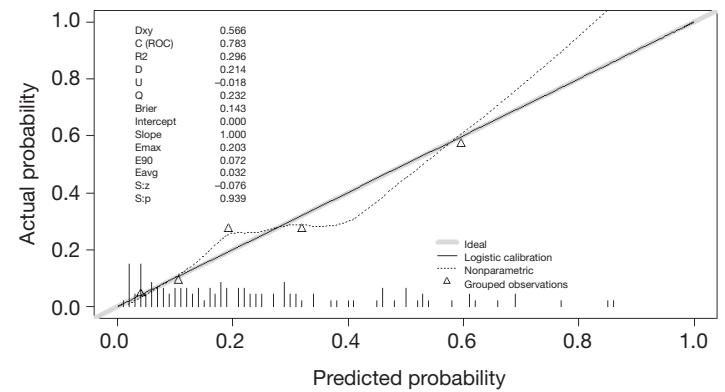

D

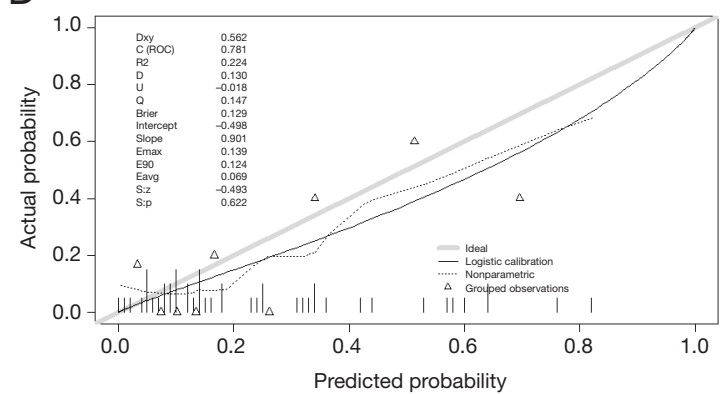

Figure 4 Receiver operating characteristic (ROC) curves and calibration curves of the three prediction models using the training cohort and the validation cohort. The ROC curves of development (A) and validation (C) of the $+\mathrm{EQS}$ model. The calibration curves of development (B) and validation $(\mathrm{D})$ of the $+\mathrm{EQS}$ model.
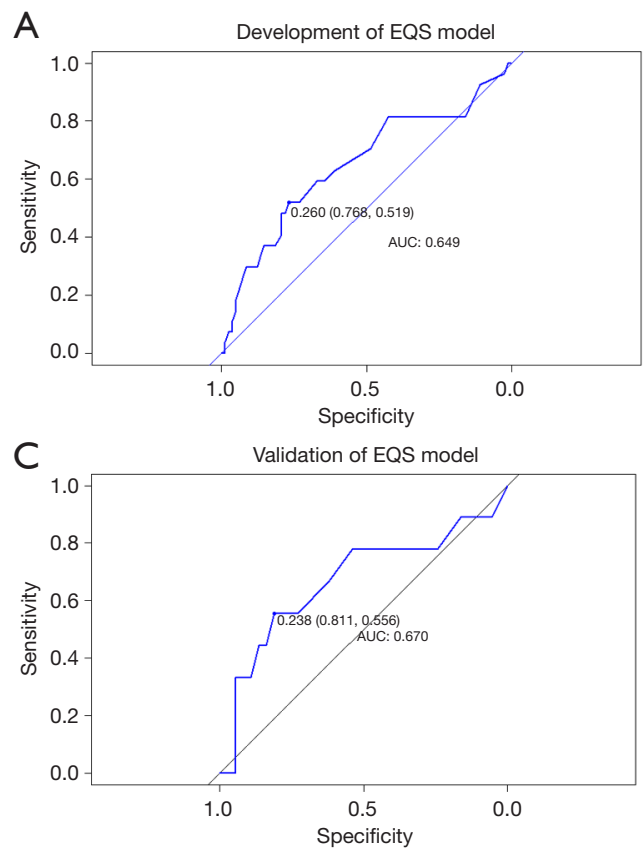

B

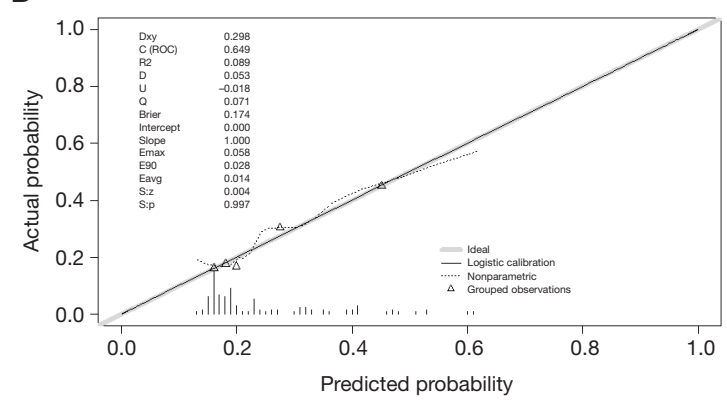

D

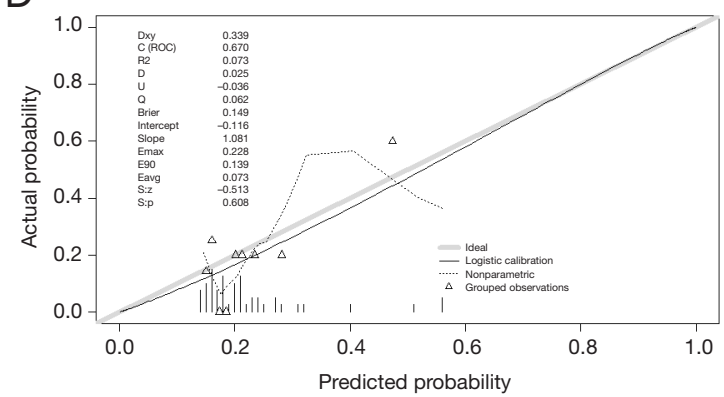

Figure 5 Receiver operating characteristic (ROC) curves and calibration curves of the three prediction models using the training cohort and the validation cohort. The ROC curves of development (A) and validation (C) of the EQS model. The calibration curves of development (B) and validation (D) of the EQS model. AUC, the area under the ROC curve. 

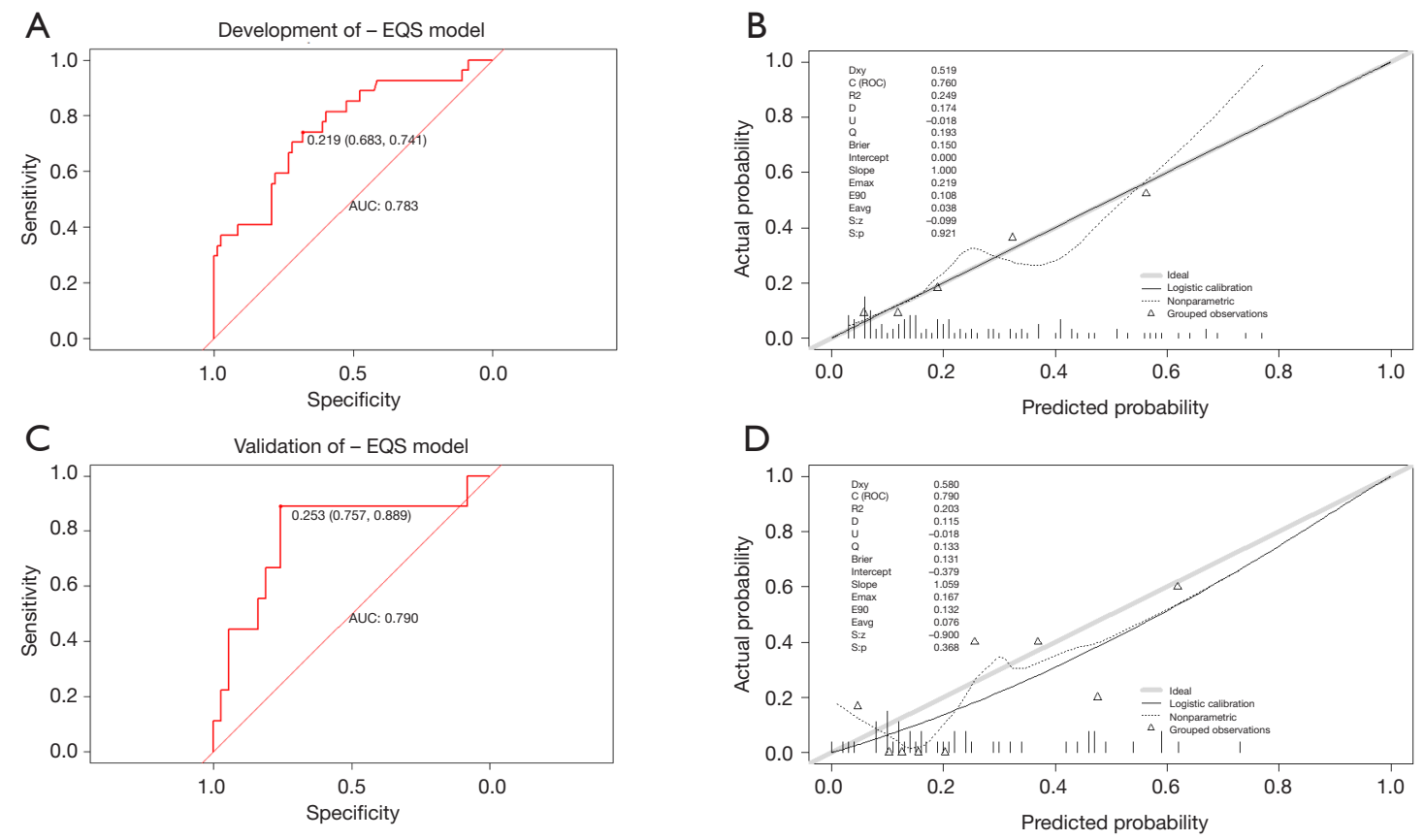

Figure 6 Receiver operating characteristic (ROC) curves and calibration curves of the three prediction models using the training cohort and the validation cohort. The ROC curves of development (A) and validation (C) of the -EQS model. The calibration curves of development (B) and validation (D) of the -EQS model. AUC, the area under the ROC curve.

proven to be predictive factors for PCa.

\section{Discussion}

The technique of targeted MP-MRI/TRUS fusion biopsy is of broad and current interest; it is supported by the joint consensus of the Society of Abdominal Radiology and the American Urological Association (36). The targeted biopsy of suspicious lesions in patients with PSA $>4 \mathrm{ng} / \mathrm{mL}$ has also been successfully performed by many other clinical applications (37-39). But a considerable number of patients with PSA in the grey zone have undergone excessive intervention. A previous study found that the majority of grey zone patients (74\%) who had been biopsied had benign lesions (5). This is similar to our observations in the present study, where $77 \%$ (119/155) of the patients had benign lesions. To reduce the number of unnecessary biopsies in the grey zone patient cohort, we established 3 cancer prediction models based on clinical features that were internally validated. Based on the evaluation of diagnostic accuracy and probability, we found that the +EQS model, which is composed of PPSA/PSA, EQS, and PI-RADS score, was reliable.
The +EQS model is different from previously proposed prediction models (15-19). This is to be expected, since such models are sensitive to differences in patient demographics, sample size, and the nature and type of independent and dependent variables included in the study design. For example, PSAD in the model by Niu et al. (15) was calculated based on MRI, but the PSAD in our model was calculated based on TRUS. This is also why we did not validate the previously reported nomograms using our validation cohort data.

The model proposed in the present study indicates that the PI-RADS score is helpful when deciding on biopsy in grey zone patients; this highlights the significance of the pre-biopsy MRI. Furthermore, the fusion of MRI and US techniques to facilitate targeted biopsy can greatly reduce the damage caused by repeated biopsy (11,40-42). However, the inter-observer consistency could affect the success of targeted MP-MRI/TRUS fusion biopsy. This study also showed that fPSA/PSA ratio is an integral predictor within the model. This is similar to the previous study by Kawamura et al. (17). However, some studies $(43,44)$ point out that PSAD or PPSA values are more useful than fPSA/PSA in the grey zone patient 


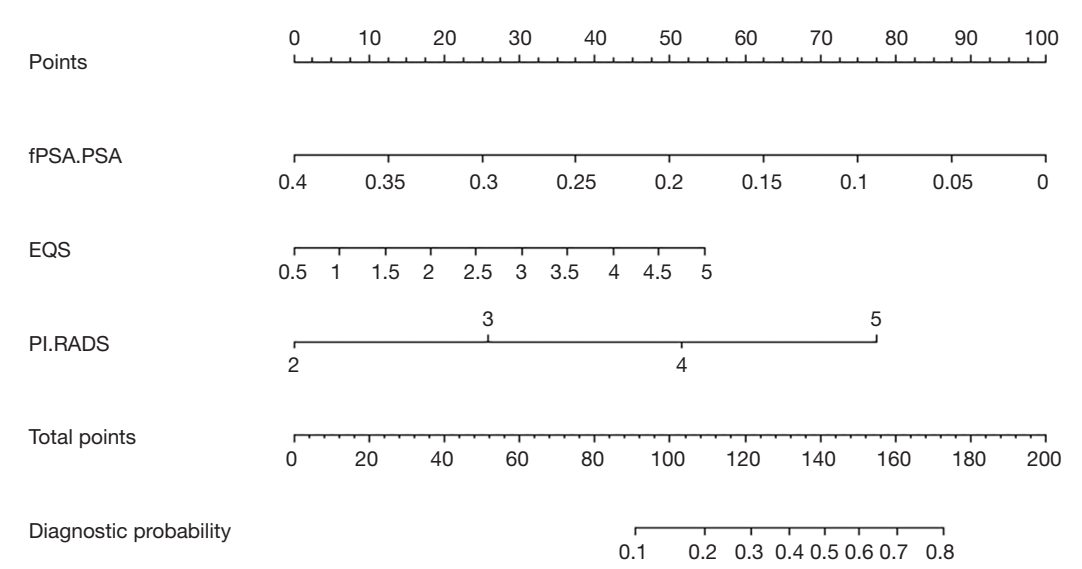

Figure 7 Nomogram based on the predictive +EQS model (fPSA/PSA, EQS, PI-RADS score) for diagnosing PCa in PSA grey zone patients. PSA, prostate-specific antigen; fPSA, free PSA; EQS, elastographic Q-analysis score; PI-RADS, prostate imaging-reporting and data system.

cohort. This is possibly because previous studies were based on serological inspection alone, or in combination with MRI findings. In the present study, the small sample size and EQS might have weakened the effects of fPSA and PSAD.

The highlight of this study is the application of elastography for improving diagnostic accuracy in grey zone patients; thereby, making full use of the advantages of US in analyzing prostate lesions. The application of elastography in prostate disease has been controversial $(28,29,45-47)$, possibly due to differences in ROI settings and probe pressure methods $(28,29)$. The World Federation for Ultrasound in Medicine and Biology (WFUMB) guidelines recommend that in the unfused state the elastic ROI should include the entire gland (48). Ding et al. $(28,29)$ chose to apply vertical targeted pressure to the location of the suspicious lesion under fusion imaging, and the ROI included just the hemilateral glands. Such targeted pressure could reduce the interference by the area of pseudosclerosis in the prostate peripheral zone, which greatly improved the diagnostic accuracy. Their results showed that the use of elastography to diagnose PCa had a threshold value of 1.95 , sensitivity of $83.5 \%$, specificity of $84.4 \%$, and an AUC of 0.870 . However, in this study, the AUC values of the EQS model were 0.649 and 0.670 in the two cohorts, and EQS made the smallest contribution in the final nomogram. While it is possible that the majority of PCa patients with PSA in the grey zone $(63 \%)$ have low GS $(3+3$ or $3+4)(49,50)$, EQS could still perform outstandingly well in diagnosing $\mathrm{PCa}$ in this patient subgroup, as indicated by the increase in the AUC value upon including EQS as a predictor during model development (-EQS model vs. +EQS model, 0.760 vs. 0.783 ). This is the first study to include EQS in a cancer prediction model for patients with PSA in the grey zone. This is significant for clinical decision-making. Additionally, elastography could complement MRI, to reduce the misdiagnoses arising from the use of single imaging modality. EQS analysis software was not only used for calculating the mean EQS, but can be used as quality control method as well. The EQS analysis curve was kept as straight as possible (fluctuation range of less than 1). In this way we standardize the quality of image to train the different operators.

The limitations of this study include those inherent to the retrospective study design, such as excluding potentially important variables [e.g., free-to-total PSA/PSAD (51)], and there may be a risk of selection bias. In addition, the sample size was small and the EQS function is specific to one instrument. We were unable to externally validate our model due to the major influence of the type of pressure method used during US. Additionally, the experienced skill of elastography is required and others' results may not be reproducted. In the future, additional data will be required to improve the internal validation of the model, or a multicenter study could be undertaken to verify the robustness of the model verify the robustness of the model it. Other parameters could be added to the model further optimize it. 


\section{Conclusions}

This study shows that pre-biopsy MRI and elastography has clinical significance for patients with prostate lesions and PSA levels in the grey zone. The nomogram based on fPSA/ PSA, EQS, and PI-RADS score can be helpful for clinical decision-making to reduce the number of unnecessary biopsies.

\section{Acknowledgments}

Funding: This work was supported by the National Natural Science Foundation of China (Grant No. 81771841) and the Guangdong Medical Science and Technology Research Fund Project (Grant No. B2019112).

\section{Footnote}

Reporting Checklist: The authors have completed the TRIPOD reporting checklist. Available at http://dx.doi. org/10.21037/tau-20-1154

Data Sharing Statement: Available at http://dx.doi. org/10.21037/tau-20-1154

Conflicts of Interest: All authors have completed the ICMJE uniform disclosure form (available at http://dx.doi. org/10.21037/tau-20-1154). The authors have no conflicts of interest to declare.

Ethical Statement: The authors are accountable for all aspects of the work in ensuring that questions related to the accuracy or integrity of any part of the work are appropriately investigated and resolved. This retrospective study was approved by the institutional review board of Shenzhen People's Hospital, and all patients provided informed consent. The study was conducted in accordance with the Declaration of Helsinki (as revised in 2013).

Open Access Statement: This is an Open Access article distributed in accordance with the Creative Commons Attribution-NonCommercial-NoDerivs 4.0 International License (CC BY-NC-ND 4.0), which permits the noncommercial replication and distribution of the article with the strict proviso that no changes or edits are made and the original work is properly cited (including links to both the formal publication through the relevant DOI and the license). See: https://creativecommons.org/licenses/by-nc-nd/4.0/.

\section{References}

1. Xiang J, Yan H, Li J, et al. Transperineal versus transrectal prostate biopsy in the diagnosis of prostate cancer: a systematic review and meta-analysis. World J Surg Oncol 2019;17:31.

2. Siegel RL, Miller KD, Jemal A. Cancer statistics. CA Cancer J Clin 2019;69:7-34.

3. Shao N, Zhu Y, Ye D. Role of polygenic hazard score in prostate-specific antigen (PSA) screening for prostate cancer. Transl Cancer Res 2018;7:S743-4.

4. Catalona WJ, Hudson MA, Scardino PT, et al. Selection of optimal prostate specific antigen cutoffs for early detection of prostate cancer: receiver operating characteristic curves. J Urol 1994;152:2037-42.

5. Stamey TA. Second Stanford conference on international standardization of prostate-specific antigen immunoassays. Urology 1995;45:173-84.

6. Welch HG, Fisher ES, Gottlieb DJ, et al. Detection of prostate cancer via biopsy in the Medicare-SEER population during the PSA era. J Natl Cancer Inst 2007;99:1395-400.

7. Graif T, Loeb S, Roehl KA, et al. Under diagnosis and over diagnosis of prostate cancer. J Urol 2007;178:88-92.

8. Singh J, Sohal SS, Lim A, et al. Cytokines expression levels from tissue, plasma or serum as promising clinical biomarkers in adenocarcinoma of the prostate: a systematic review of recent findings. Ann Transl Med 2019;7:245.

9. Shoji S. Magnetic resonance imaging-transrectal ultrasound fusion image-guided prostate biopsy: Current status of the cancer detection and the prospects of tailormade medicine of the prostate cancer. Investig Clin Urol 2019;60:4-13.

10. Shah PH, Patel VR, Moreira DM, et al. Implementation of multiparametric magnetic resonance imaging technology for evaluation of patients with suspicion for prostate cancer in the clinical practice setting. BJU Int 2019;123:239-45.

11. Yarlagadda VK, Lai WS, Gordetsky JB, et al. MRI/US fusion-guided prostate biopsy allows for equivalent cancer detection with significantly fewer needle cores in biopsynaive men. Diagn Interv Radiol 2018;24:115-20.

12. Mendhiratta N, Rosenkrantz AB, Meng X, et al. Magnetic resonance imaging-ultrasound fusion-targeted prostate biopsy in a consecutive cohort of men with no previous biopsy: reduction of over-detection through improved risk stratification. J Urol 2015;194:1601-6.

13. Mottet N, van der Bergh RCN, Briers E, et al. European 
Association of Urology prostate cancer guidelines. Available online: http://uroweb.org/guideline/prostatecancer/.

14. Hwang SI, Lee HJ, Lee SE, et al. Value of MR-US fusion in guidance of repeated prostate biopsy in men with PSA $<10$ ng/mL. Clin Imaging 2019;53:1-5.

15. Niu XK, Li J, Das SK, et al. Developing a nomogram based on multiparametric magnetic resonance imaging for forecasting high-grade prostate cancer to reduce unnecessary biopsies within the prostate-specific antigen gray zone. BMC Medical Imaging 2017;17:11.

16. Fang D, Ren D, Zhao C, et al. Prevalence and risk factors of prostate cancer in Chinese Men with PSA 4-10 ng/mL who underwent TRUS-guided prostate biopsy: the utilization of PAMD score. Biomed Res Int 2015;2015:596797.

17. Kawamura K, Suzuki H, Kamiya N, et al. Development of a new nomogram for predicting the probability of a positive initial prostate biopsy in Japanese patients with serum PSA levels less than 10ng/mL. Int J Urol 2008;15: 598-603.

18. Zaytoun OM, Kattan MW, Moussa AS, et al. Development of Improved Nomogram for Prediction of Outcome of Initial Prostate Biopsy Using Readily Available Clinical Information. Urology 2011;78:392-8.

19. Jeong IG, Lim JH, Kim SC, et al. Nomogram using transrectal ultrasound-derived information predicting the detection of high grade prostate cancer on initial biopsy. Prostate International 2013;1:69-75.

20. Panebianco V, Giganti F, Kitzing YX, et al. An update of pitfalls in prostate mpMRI: a practical approach through the lens of PI-RADS v. 2 guidelines. Insights Imaging 2018;9:87-101.

21. Bjurlin MA, Renson A, Rais-Bahrami S, et al. Predicting Benign Prostate Pathology on Magnetic Resonance Imaging/Ultrasound Fusion Biopsy in Men with a Prior Negative 12-core Systematic Biopsy: External Validation of a Prognostic Nomogram. Eur Urol Focus 2019;5:815-22.

22. Sonn GA, Fan RE, Ghanouni P, et al. Prostate magnetic resonance imaging interpretation varies substantially across radiologists. Eur Urol Focus 2019;5:592-9.

23. Muller BG, Shih JH, Sankineni S, et al. Prostate cancer: interobserver agreement and accuracy with the revised Prostate Imaging Reporting and Data System at multiparametric MR imaging. Radiology 2015;277:741-50.

24. Tyloch DJ, Tyloch JF, Adamowicz J, et al. Elastography in prostate gland imaging and prostate cancer detection. Med
Ultrason 2018;20:515-23.

25. Aboumarzouk OM, Ogston S, Huang Z, et al. Diagnostic accuracy of transrectal elastosonography (TRES) imaging for the diagnosis of prostate cancer: a systematic review and meta-analysis. BJU Int 2012;110:1414-23.

26. Sang L, Wang XM, Xu DY, et al. Accuracy of shear wave elastography for the diagnosis of prostate cancer: A metaanalysis. Sci Rep 2017;7:1949.

27. Woo S, Suh CH, Kim SY, et al. Shear-Wave Elastography for Detection of Prostate Cancer: A Systematic Review and Diagnostic Meta-Analysis. AJR Am J Roentgenol 2017;209:806-14.

28. Ding Z, Jiao $\mathrm{Y}, \mathrm{Wu} \mathrm{H}$, et al. Clinical value of the elastographic q-analysis score in assisting real-time elastography-guided prostate biopsy: a retrospective study of 125 patients. J Ultrasound Med 2020;39:83-7.

29. Ding Z, Ye X, Zhang L, et al. Evaluation of the Performance of the Ultrasound (US) Elastographic Q-Analysis Score Combined With the Prostate Imaging Reporting and Data System for Malignancy Risk Stratification in Prostate Nodules Based on Transrectal US-Magnetic Resonance Imaging Fusion Imaging. J Ultrasound Med 2019;38:2991-8.

30. Ophir J, Céspedes I, Ponnekanti H, et al. Elastography: a quantitative method for imaging the elasticity of biological tissues. Ultrason Imaging 1991;13:111-34.

31. Brock M, von Bodman C, Palisaar RJ, et al. The impact of real-time elastography guiding a systematic prostate biopsy to improve cancer detection rate: A prospective study of 353 patients. J Urol 2012;187:2039-43.

32. Weinreb JC, Barentsz JO, Choyke PL, et al. PI-RADS Prostate Imaging-Reporting and Data System: 2015, version 2. Eur Urol 2016;69:16-40.

33. Tibshirani R. The lasso method for variable selection in the Cox model. Stat Med 1997;16: 385-95.

34. Jin X, Ai Y, Zhang J, et al. Noninvasive prediction of lymph node status for patients with early-stage cervical cancer based on radiomics features from ultrasound images. Eur Radiol 2020;30:4117-24.

35. Cleveland WS, Grosse E, Shyu WM. In: Chambers JM, Hastie TJ. editors, Statistical models in S, Vol II, Local regression models. New York, 1992.

36. Rosenkrantz AB, Verma S, Choyke P, et al. Prostate magnetic resonance imaging and magnetic resonance imaging targeted biopsy in patients with a prior negative biopsy: a consensus statement by AUA and SAR. J Urol 2016;196:1613-8.

37. Westhoff N, Siegel F, Peter C, et al. Defining the target 
prior to prostate fusion biopsy: the effect of MRI reporting on cancer detection. World J Urol 2019;37:327-35.

38. Hakozaki Y, Matsushima H, Murata T, et al. Detection rate of clinically significant prostate cancer in magnetic resonance imaging and ultrasonography-fusion transperineal targeted biopsy for lesions with a prostate imaging reporting and data system version 2 score of 3-5. Int J Urol 2019;26:217-22.

39. Kim YJ, Huh JS, Park KK. Effectiveness of Bi-Parametric MR/US Fusion Biopsy for Detecting Clinically Significant Prostate Cancer in Prostate Biopsy Naive Men. Yonsei Med J 2019;60:346-51.

40. Delongchamps NB, Peyromaure M, Schull A, et al. Prebiopsy magnetic resonance imaging and prostate cancer detection: comparison of random and targeted biopsies. J Urol 2013;189:493-9.

41. Puech P, Rouviã-Re O, Renard-Penna R, et al. Prostate cancer diagnosis: multiparametric MR-targeted biopsy with cognitive and transrectal US-MR fusion guidance versus systematic biopsy--prospective multicenter study. Radiology 2013;268:461-9.

42. Siddiqui MM, Raisbahrami S, Turkbey B, et al. Comparison of MR/ultrasound fusion-guided biopsy with ultrasoundguided biopsy for the diagnosis of prostate cancer. JAMA 2015;313:390-7.

43. Catalona WJ, Partin AW, Slawin KM, et al. Use of the percentage of free prostate-specific antigen to enhance differentiation of prostate cancer from benign prostatic disease: a prospective multicenter clinical trial. JAMA 1998;279:1542-7.

44. Zheng XY, Xie LP, Wang YY, et al. The use of prostate

Cite this article as: Ding $\mathrm{Z}, \mathrm{Wu} \mathrm{H}$, Song D, Tian H, Ye X, Liang W, Jiao Y, Hu J, Xu J, Dong F. Development and validation of a nomogram for predicting prostate cancer in men with prostate-specific antigen grey zone based on retrospective analysis of clinical and multi-parameter magnetic resonance imaging/transrectal ultrasound fusion-derived data. Transl Androl Urol 2020;9(5):2179-2191. doi:10.21037/tau-20-1154 specific antigen (PSA) density in detecting prostate cancer in Chinese men with PSA levels of 4-10 ng/mL. J Cancer Res Clin Oncol 2008;134:1207-10.

45. Tu X, Qiu S, Chang T, et al. The role of real-time elastography- targeted biopsy in the detection and diagnosis of prostate cancer: A systematic review and metaanalysis. Medicine (Baltimore) 2018;97:e0220.

46. Teng J, Chen M, Gao Y, et al. Transrectal sonoelastography in the detection of prostate cancers: a meta-analysis. BJU Int 2012;110:E614-20.

47. Zhang B, Ma X, Zhan W, et al. Real-time elastography in the diagnosis of patients suspected of having prostate cancer: a meta-analysis. Ultrasound Med Biol 2014;40:1400-7.

48. Barr RG, Cosgrove D, Brock M, et al. WFUMB Guidelines and Recommendations on the Clinical Use of Ultrasound Elastography: Part 5. Prostate. Ultrasound Med Biol 2017;43:27-48.

49. Aigner F, Schafer G, Steiner E, et al. Value of enhanced transrectal ultrasound targeted biopsy for prostate cancer diagnosis: a retrospective data analysis. World J Urol 2012;30:341-6.

50. Junker D, Schafer G, Aigner F, et al. Potentials and limitations of real-time elastography for prostate cancer detection: A whole-mount step section analysis. ScientificWorldJournal 2012;2012:193213.

51. Nan LB, Yin XT, Gao JP. Significant Diagnostic Value of Free-Serum PSA (FPSA)/Prostate-Specific Antigen Density (PSAD) and (F/T)/PSAD for Prostate Cancer of the Chinese Population in a Single Institution. Med Sci Monit 2019;25:8345-51. 